\title{
Classification performance assessment in side scan sonar image while underwater target object recognition using random forest classifier and support vector machine
}

\author{
R. Kumudham ${ }^{1 *}$, V. Rajendran ${ }^{2}$ \\ ${ }^{I}$ Department of ECE, School of Engineering, Vels Institute of Science, Technology and Advanced Studies (VISTAS), Chennai. \\ ${ }^{2}$ Department of ECE, School of Engineering, Vels Institute of Science, Technology and Advanced Studies (VISTAS), Chennai. \\ *Corresponding author E-mail: kumudham.sree@gmail.com
}

\begin{abstract}
Ocean mine have been a major threat to the safety of vessels and human lives for many years. Identification of mine-like objects is a pressing need for military, and other ocean meets. In mine, countermeasures operations, sonar equipment are utilised to detect and classify mine- like objects if their sonar signatures are similar to known signatures of mines. The classification of underwater mines is an important task, for the safety of ports, harbors and the open sea. Mine detection is needed in military applications because it has been a threat to many lives and vessels. Although the task of finding mine like objects has received recent attention, very little has been published on the problem of discriminating mine-like (target) objects (MLO) and non-mine like (target) objects of similar size and shape. This paper deals with the recognition of mine like and non mine like objects. The recognition is done through robust Random Forest technique.
\end{abstract}

Keywords: Underwater mine, SONAR image, side scan sonar image, random forest classifier, mine like object detection, non mine like object detection.

\section{Introduction}

Mine is an explosive device which causes destruction to submarines or ships. This has been buried underneath water, when an enemy ship crosses, the mine explodes. Mine detection is needed in military applications because it has been a threat to many lives and vessels. Ocean mine have been a major threat to the safety of vessels and human lives for many years. Identification of mine-like objects is a pressing need for military, and other ocean meets. In mine, countermeasures operations, side scan sonar are used to detect and classify mine- like target objects if their sonar signatures are similar to known signatures of mines. Very little research has been done on the problem of detecting non-mine objects and mine-like objects (MLO). It is difficult for the human operators to detect the mines and through images captured by side scan sonar equipment the work has been made easier. Automated system for target recognition (ATR), applied to mine classification solves issues involved in underwater mine recognition. The proposed method mainly concentrates with selection of feature and recognition of mine like and non mine like objects. The features are selected using D-SFFS method (Extension of Sequential Floating Forward Selection). The selected features are classified as mine and non mine using Random Forest Algorithm.

\section{LiterAture survey}

Tai Fei, D.Kraus, A.M.Zoubir [1][12] concentrated on object classification and feature selection and provided several contributions for automated system for target recognition, applied to mine classification in underwater. First, the filter method is applied for the feature selection. The authors used novel

composite relevance measure (CRM) which combined several measures. It provided an assessment of both combinations of the linear and nonlinear features. A learning scheme with the Dempster-Shafer theory is used to combine the output obtained by different classification methods. This method improved the performance of the classification.

Bryan Thompson, Jered Cartmill, Mahmood R. Azimi-Sadjadi, and Steven G. Schock [2] examined fusion classifiers which is CCA based. The authors extracted two features CCA (canonical correlation analysis )/MCCA(Multichannel canonical correlation analysis) features form the data collected by Boss system- buried objects scanning sonar system. Classifiers results are produced for both the CCA and MCCA features. The results proved that MCCA features yielded better result than CCA features.

Kenneth Stewart, Min Jiang, and Martin Marra [3] proposed Back propagation neural network Classification. During the process, the information passes to output layer, through hidden layers, from the input layer. The datas are collected from geoacoustic provinces of sediment pond, ridge flank, axial valley. The authors obtained the classification accuracy rates as follows for valleys, $80.1 \%$, sediment ponds, $85 \%$, ridge flanks, $91 \%$.

Anthony R. Castellano Brian C. Gray [4] proposed Neural network algorithm for classification, signal detection and feature is extracted using SSS geometry. The authors worked on real time data identifying bottom objects.

The signature of the target appearance changes slightly according to operational and environmental conditions. The specific parameters such as elevation, range, transmitted pulse duration, bandwidth, frequency, sampling frequency, and grazing angle can be deduced from side scan images. In practical situation, the problems of target classification in underwater depends on the 
size, shape, orientation, the position of the target such as suspended, bottom, or buried in the water column relative to the seabed and its type.

JoEllen Wilbur, Robert J. McDonald and Jason stack [5] used kernel based classifiers. Contourlet transform modelled the ridges and contours in the image effectively. The structure (lattice) divides the image into multiple scales. Each scale is then mosaiced into contour partitions. Contourlet coefficients are obtained along the ridges and contours of the image from the contour segments.

Kumudham. R, Rajendran.V [22] classified the objects in seabed using the reflectance properties of the different materials and there by classifying as rock, clay, etc based on the nature of the material.
The existing method involves object recognition using support vector machine by extracting Gray Level Co occurrence Matrix features [17].

\section{Proposed work}

The proposed work involves recognition of mine like (target) objects and non mine like (target) objects from sonar images. The figure 1 given below gives the flow of the recognition of the mine like objects and it is further classified as truncated cone, stone-non mine like object and cylinder. The steps are as follows.

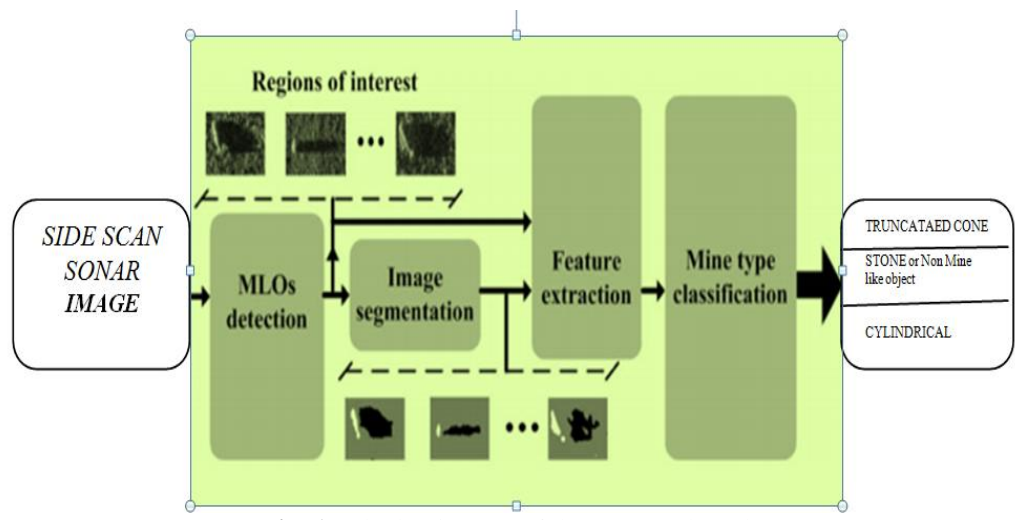

Fig. 1: Block Diagram of the proposed work

Sidescan sonar images are acquired from the database of EDGETECH. The images may have or may not have mine like objects. The block diagram of the proposed method, an Automated Target Recognition system is depicted in Fig 1. The input is side scan sonar image, and the output is the Mine like Object detection (a) a cylindrical mines, (b) a truncated cone mines, and non mine like object detection, stones. The original side scan sonar image is preprocessed and morphological processed image is obtained. The region of interest (ROI) and non ROI part is obtained, for this a labeling algorithm is included in order to preserve boundaries corresponding to each area (target or seabed). The region of interests are obtained and forwarded to the next step, i.e., the segmentation and the feature selection. The aim of feature extraction is to select the inputs for the mine-type recognition. In the proposed method features are selected using SFFS method (Extension of Sequential Floating Forward Selection).

The algorithm for SFFS is as follows:

1. $\mathrm{z}=\varnothing$

2. Select the feature (best) $\mathrm{y}+=\arg \max \mathrm{y} \notin \mathrm{z} k J(\mathrm{z} k+) \mathrm{z} k=\mathrm{z} k$ $+\mathrm{Z}+; k=k+1$

3. Select the feature (worst) $* \mathrm{y}-=\arg \max \mathrm{y} \in \mathrm{z} k J \mathrm{z} k-x 4$.

If $J \mathrm{z} k-\mathrm{y}->J \mathrm{z} k$ then $\mathrm{z} k+1=\mathrm{z} k-\mathrm{y}-; k=k+1$ Go to step 3 Else Go to step 2 .

The selected features are input to the Random Forest classifier to recognise the object as mine or non mine. Random Forest algorithm an ensemble classifier, proposed by Leo Breiman [6] has many decision trees. A training set is generated first for each tree in the forest, by randomly choosing $\mathrm{M}$ times from the original data samples, and the remaining samples are utilised as test set. The number of feature used for dividing each node of decision tree $(n)$ is mentioned first when each node of single decision tree is dividing. Then $n$ out of $N$ features are selected randomly and the best characteristics on these $N$ features is used to divide the node, such that at each node of single decision tree the impurity is minimised and each tree grows without cutting. A Random Forest with $t$ decision trees is formed by repeating $t$ times as above procedure, and then the Random Forest is used to predict test data. The final classification results are decided. Random Forest has two most important parameters, one is the number of trees $(\mathrm{k})$, another parameter is the number of features used for divide each node of decision tree $(\mathrm{n}, \mathrm{n} \ll \mathrm{N}$ where $\mathrm{N}$ is the total number of features). The advantage of this method is that it doesn't produce over fitting when the high dimensional data is used.

\section{Results}

The following Fig 2 illustrates the original image; RGB converted image, enhanced image features as well as the morphological feature enhanced image views with binary nature.

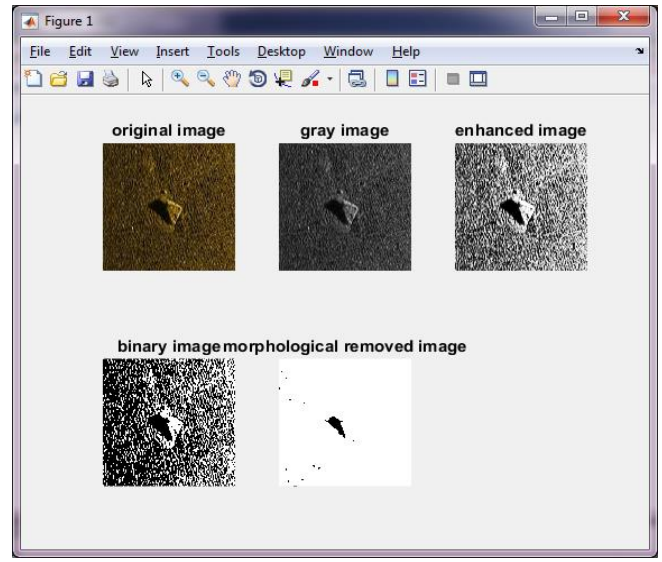

Fig.2: Binary image- morphological removed image

The following Fig 3 illustrates the clear view of Region-of-Interest portion as well as the Non-Region-of-Interest (Non-ROI) portions separately. 


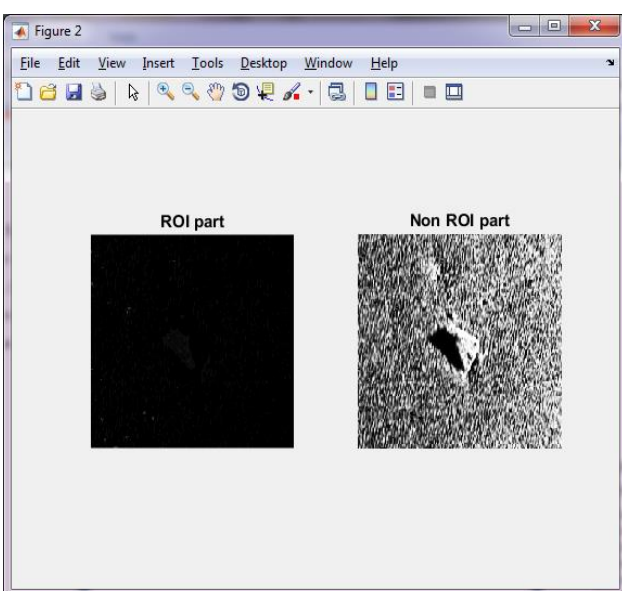

Fig.3: ROI Part and non - ROI part

The following figure illustrates the clear perception of clarified border and the enhanced view of regions.

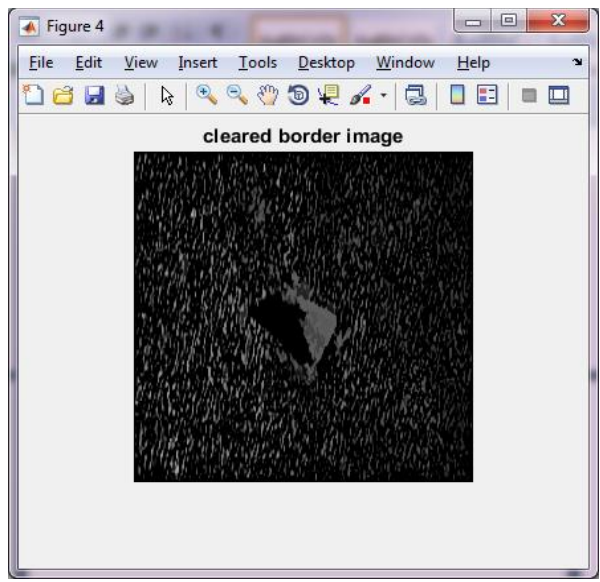

Fig. 4: Cleared border image

The following figure illustrates the segmented portion of the mine objects and highlights the portion with contrast enhancements.

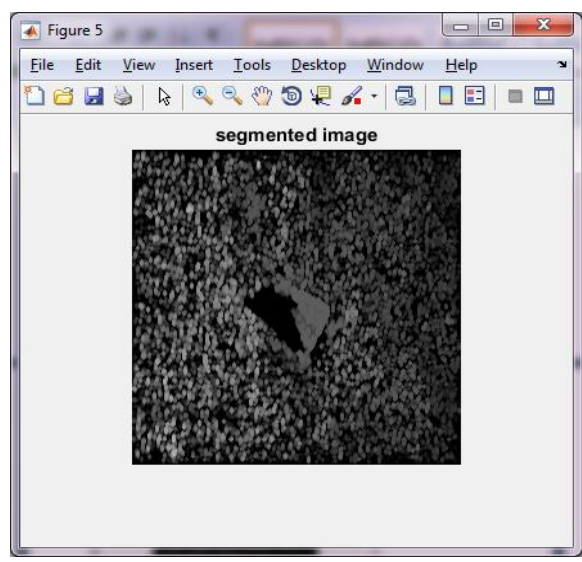

Fig.5: Segmented image

The Fig 6 illustrates the smoothening portion for a distance image to both left and right side view as well as the message boxes are illustrated the identified object type and result view of the original image.

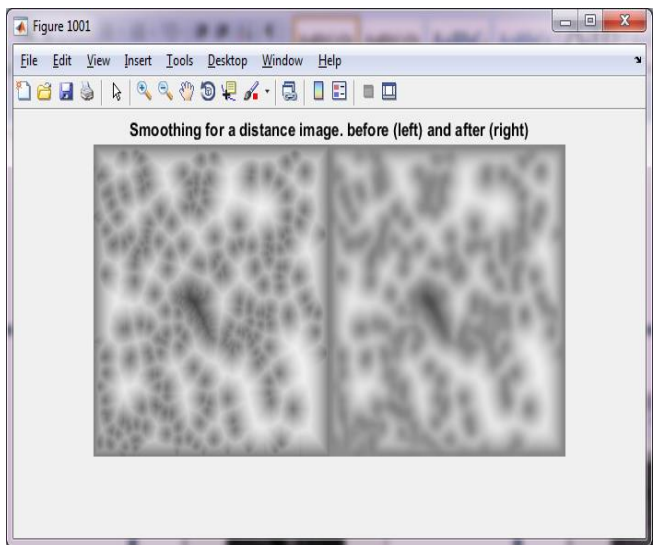

Fig.6: Smoothing for a distance Image Before (Left) and After (Right).

Fig 7 and fig 8 illustrates the detection of mine like object and further detection of mine as truncated cone.

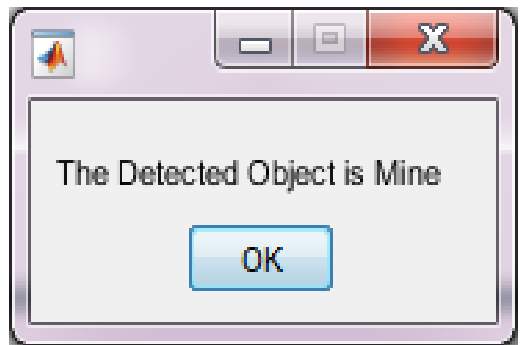

Fig.7: Mine like object detection

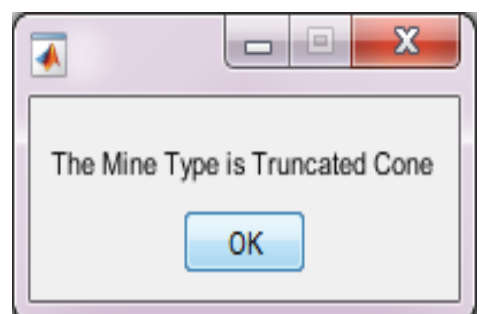

Fig.8: Mine like object detection-truncated cone

The following Fig 8 illustrates the given object is a mine and further detected as cylindical mine.
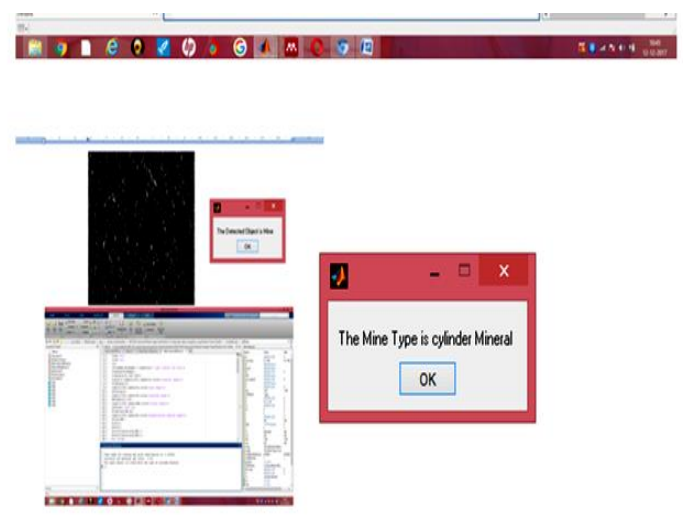

Fig.7: Cylindrical mine

The following Fig 8 and Fig 9 illustrates the given object is not a mine and it has been detected as stone. 


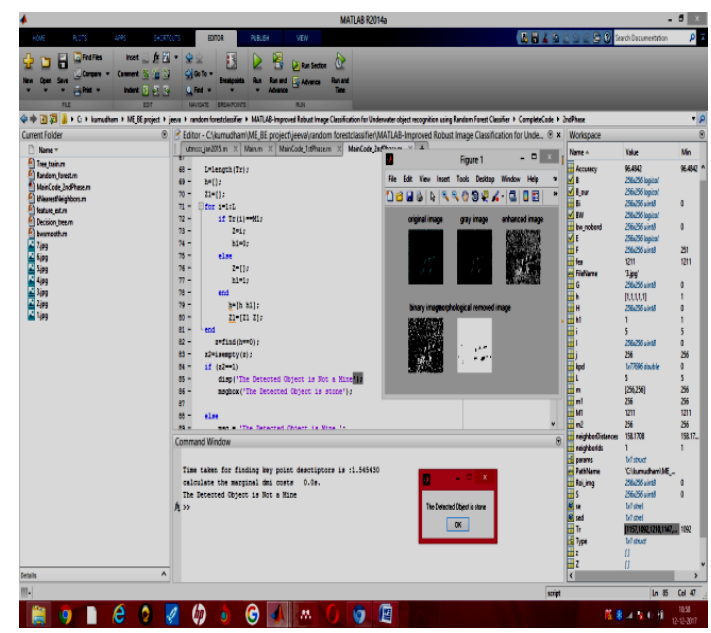

Fig.8: Non Mine like object detection (Stone)

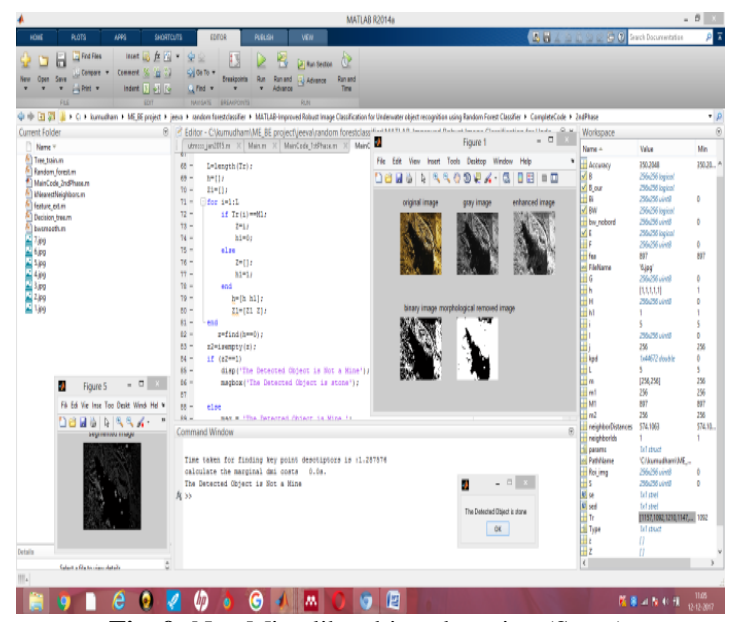

Fig. 9: Non Mine like object detection (Stone)

Classification Results

The performance of the proposed method is assessed by considering the classification accuracy, which is quantified by the empirical classification rate

$$
\rho=\mathrm{mCorrect} / \mathrm{M}
$$

Where' mcorrect' is the number of target objects whose classification results are accurate in comparison to ground truth[1].

Table 1: Classification rate $(\rho)$

\begin{tabular}{|c|c|c|c|}
\hline S.No & Sonar Image & $\begin{array}{l}\text { Random Forest } \\
\text { Algorithm }\end{array}$ & $\begin{array}{l}\text { Support Vector } \\
\text { Machine }\end{array}$ \\
\hline 1 & & 0.9667 & 0.7962 \\
\hline 2 & & 0.9762 & 0.8000 \\
\hline 3 & 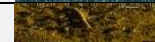 & 0.9810 & 0.8571 \\
\hline 4 & & 0.8967 & 0.7896 \\
\hline
\end{tabular}

\section{Conclusion and future work}

In the present work, the object in underwater is recognized as mine and non mine using robust random classifier and further classified mine like object as truncated cone, cylindrical mine. The proposed method is compared with the existing method of support vector machine classifier. The Random forest algorithm provided better classification rate than support vector machine for mine classification. The future work is to detect mine and non mine like object in real time applications as well as to find the best classifiers algorithm that works well in the huge volume side scan sonar image data.

\section{References}

[1] Tai F, Kraus D \& Zoubir AM, "Contributions to Automatic Target Recognition Systems for Underwater Mine Classification”, IEEE Transactions on Geo science and Remote Sensing, (2015).

[2] Bryan DT, Jered C, Mahmood RA \& Schock SG, "A Multichannel Canonical Correlation Analysis Feature Extraction with Application to Buried Underwater Target Classification", IEEE International Joint Conference on Neural Network Proceedings, (2006).

[3] Stewart WK, Min J \& Marra M, "A neural network approach to classification of sidescan sonar imagery from a midocean ridge area", IEEE Journal of Oceanic Engineering (1994).

[4] Castellano AR \& Gray BC, "Autonomous interpretation of side scan sonar returns", Symposium on Autonomous Underwater Vehicle Technology, 1990.

[5] JoEllen W \& Robert JM \& Jason S, "Contourlet Detection and Feature Extraction for Automatic Target Recognition", IEEE International Conference on Systems, Man, and Cybernetics San Antonio, (2009).

[6] Breiman L, "Random Forests", Machine Learning, Vol.45, (2001), pp. 5-32.

[7] Johnson S \& Deaett A, "The application of automated recognition techniques to side-scan sonar imagery", IEEE J. Ocean. Eng., Vol.19, No.1, (1994), pp.138-144.

[8] Henriksen L, "Real-time underwater object detection based on an electricallyscanned high-resolution sonar", In Proc. Symp. Auton. UnderwaterVeh. Technol., (1994), pp.99-104.

[9] Fandos R. \& Zoubir AM, "Optimal feature set for automaticdetection and classification of underwater objects in SAS images", IEEE J. Sel. Topics Signal Process., Vol.5, No.3, (2011), pp.454-468.

[10] Piper JE, Lim R, Thorsos EI \& Williams KL, "Buried spheredetection using a synthetic aperture sonar", IEEE J. Ocean. Eng., Vol.34, No.4, (2009), pp.485-494.

[11] Hayes M \& Gough P, "Synthetic aperture sonar: A review ofcurrent status", IEEE J. Ocean. Eng., Vol.34, No.3, (2009), pp. 207-224.

[12] Piper J, Commander K, Thorsos E \& Williams K, "Detection of buried targets using a synthetic aperture sonar", IEEE J. Ocean. Eng., Vol.27, No.3, (2002), pp.495-504.

[13] Fandos R, Zoubir AM \& Siantidis K, "Unified design of a featurebased ADAC system for mine hunting using synthetic aperturesonar", IEEE Trans. Geosci. Remote Sens., Vol.52, No.5, (2014). pp.2413-2426.

[14] Myers V. \& Williams D, "Adaptive multiview target classification insynthetic aperture sonar images using a partially observable Markovdecision process", IEEE J. Ocean. Eng., Vol.37, No.1, (2012), pp.45-55.

[15] Fei T. \& Kraus D, "An expectation-maximization approach assisted byD empster Shafer theory and its application to sonar image segmentation", in Proc. IEEE ICASSP, (2012), pp.11611164.

[16] Fandos R \& Zoubir AM, "Enhanced initialization scheme for a three region Markovian segmentation algorithm and its application to SAS images", Proc. 10th ECUA, Vol.3 (2010), pp.1323- 1331.

[17] Kumudham R \& Rajendran V, "Object recognition in underwater sonar images using support vector machine", International Journal of Control Theory and Applications (IJCTA), Vol.10, No. 32, (2017), pp.283-290.

[18] Reed S, Petillot Y \& Bell J, "An automatic approach to the detectionand extraction of mine features in sidescan sonar", IEEE J. Ocean. Eng., Vol.28, No.1, (2003), pp.90-105.

[19] Yang M, Kpalma K \& Ronsin J, "A Survey of Shape Feature Extraction Techniques", InTech, Vol.3 (2008), pp.43-90.

[20] Tang Y, Li B, Ma H \& Lin J, "Ring-projection-wavelet-fractal signatures:A novel approach to feature extraction", IEEE Trans. CircuitsSyst. II, Analog Digit. Signal Process., Vol.45, No.8, (1998), pp.1130-1134. 
[21] Weszka JS, C. R. Dyer, and A. Rosenfeld, "A comparative study of texture measures for terrain classification," IEEE Trans. Syst., Man, Cybern.,vol. SMC-6, No.4, pp. 269-285, (1976).

[22] Kumudham R \& Rajendran V, "Side scan sonar image denoising and classification", Journal of Advanced Research in Dynamical \& Control Systems, (2017), pp.55-65.

[23] Kumudham R \& Rajendran V, "Implementation of various segmentation algorithms on side scan sonar images and analysing its performance", ARPN, Vol.12, No.8, (2017), pp.2396-2400.

[24] Kumudham R \& Rajendran V, "Speeded up robust feature extraction from underwater sonar images", International Journal of Control Theory and Appplications (IJCTA), Vol.10, No.32, (2017), pp.277-282.

[25] Kumudham R Dhanalakshmi ASG, \& Rajendran V, "Comparison of The Performance Metrics of Median Filter and Wavelet Filter when applied on Sonar Images for Denoising", IEEE sponsored International Conference on Computation of Power Energy Information and Communication, (2016). 